\title{
FOLIA
}

Amazónica

Revista del Instituto de Investigaciones

de la Amazonía Peruana

\section{PRIMER REGISTRO DE INGESTIÓN DE MICROPLÁSTICOS POR UN PEZ DE IMPORTANCIA COMERCIAL EN LA CIUDAD DE IQUITOS, AMAZONÍA PERUANA}

\author{
Werner CHOTA-MACUYAMA ${ }^{1, *}$, Jhancarlo CHONG MENDOZA ${ }^{1}$ \\ 1 Instituto de Investigaciones de la Amazonía Peruana - IIAP. Dirección de Investigación en \\ Ecosistemas Acuáticos Amazónicos. Laboratorio de Calidad de Agua; Carretera lquitos-Nauta \\ km 4.5, San Juan Bautista, Maynas, Loreto, Perú. \\ * Correo electrónico: wernerchotam@yahoo.com
}

\begin{abstract}
RESUMEN
El estudio reporta por primera vez para la Amazonía peruana la ingestión de microplásticos en un pez de importancia económica, registrados en individuos provenientes del Mercado Belén de la ciudad de Iquitos, región Loreto, Perú. De las once especies analizadas (50 individuos), sólo en seis individuos de la especie boquichico Prochilodus nigricans Agassiz, 1829 fueron registrados en total 17 partículas de microplásticos, con un promedio (y desviación estándar) de $0,34( \pm 1,19)$ microplásticos por individuo. La frecuencia de ocurrencia de las partículas de microplásticos para los 50 individuos fue $12 \%$ y para $P$. nigricans fue $46,2 \%$. Los tamaños estuvieron en un rango de 0,40 a $4,39 \mathrm{~mm}$ y las formas encontradas fueron los filamentos (14) y los fragmentos irregulares (3). De los colores registrados, el azul se presentó en mayor número (10 microplásticos), seguido del rojo (5 microplásticos), verde (1 microplástico) y celeste (1 microplástico). Los resultados estarían sugiriendo que algunas zonas de pesca están siendo afectadas por la mala gestión de los residuos plásticos impactando de forma negativa en los ecosistemas y la ictiofauna de la Amazonía peruana; y a través del consumo podría afectar a la salud de las personas.
\end{abstract}

PALABRAS CLAVE: Amazonía, plástico, polución, residuos sólidos, río Itaya. 


\title{
FIRST RECORD OF MICROPLASTIC INGESTION BY AN IMPORTANT COMMERCIAL FISH IN THE CITY OF IQUITOS, PERUVIAN AMAZON
}

\begin{abstract}
This study reports for the first time the microplastics ingestion in a important commercial fish in the Peruvian Amazon, recorded in individuals from the Belén market in the city of Iquitos, Loreto region, Peru. Of the eleven species analyzed (50 individuals), only in six individuals of boquichico Prochilodus nigricans Agassiz, 1829 were recorded 17 microplastic particles with an average (and standard deviation) of $0.34( \pm 1.19)$ microplastics per individual. The frequency of occurrence of microplastic particles for the 50 individuals was 12\%, and for $P$. nigricans was $46.2 \%$. The sizes ranged from 0.40 to $4.39 \mathrm{~mm}$ and the shapes found were filaments (14 microplastics) and irregular fragments (3 microplastics). In the colors, blue was present in greater numbers (10 microplastics), followed by red (5 microplastics), green (1 microplastic) and light blue (1 microplastic). The results would be suggesting that some fishing areas are being affected by the mismanagement of plastic waste; negatively impacting the ecosystems and the ichthyofauna of the Peruvian Amazon, and through consumption could affect people's health.
\end{abstract}

KEYWORDS: Amazon, plastic, pollution, solid waste, Itaya river. 


\section{INTRODUCCIÓN}

La producción cada vez mayor de plásticos y su vida útil relativamente corta, combinada con malas prácticas de eliminación de desechos $\mathrm{y}$ vertidos accidentales, han dado lugar a la acumulación de este material en ambientes acuáticos en todo el mundo. Esta situación es preocupante por el largo tiempo de degradación del plástico y la posibilidad de que sea ingerido por organismos acuáticos (De Sales Ribeiro et al., 2020). Los microplásticos son cualquier partícula sólida sintética o matriz polimérica, de forma regular o irregular y con un tamaño que varía de $1 \mu \mathrm{m}$ a $5 \mathrm{~mm}$, de origen de fabricación primario o secundario que son insolubles en agua (Frias \& Nash, 2019). El plástico de origen primario se refiere a que los microplásticos fueron producidos en dimensiones microscópicas, y secundario que los microplásticos resultan de procesos de degradación y fragmentación en el ambiente (Cole et al., 2011). Las bolsas de plástico, las botellas, los aparejos de pesca, y otros productos están entrando en los cuerpos de agua amazónicos y se degradan en partículas meso y microplásticas que pueden ser ingeridas, directa o indirectamente por los peces a través de la cadena alimenticia (Andrade et al., 2019). Por tanto, el pescado y los productos pesqueros pueden estar contaminados con microplásticos y sus contaminantes asociados, impactando en la seguridad alimentaria y la salud humana. Es por ello que en la última década la contaminación por microplásticos ha recibido mayor atención por la comunidad científica, organizaciones internacionales, gobiernos y medios públicos, impulsado principalmente por preocupaciones ambientales y de salud humana. (Lusher et al., 2017)

La contaminación por microplásticos está muy extendida en los ecosistemas marinos y constituye una gran amenaza para la biodiversidad. Sin embargo, nuestro conocimiento de los impactos de los microplásticos en los ambientes de agua dulce y la biota es todavía muy limitado (SilvaCavalcanti et al., 2017; Urbanski et al., 2020). El primer reporte de microplásticos para la Amazonía fue realizado en Brasil en 16 especies de serrasalmidos en el año 2019 (Andrade et al., 2019). En otras especies de agua dulce fue reportado en Prochilodus lineatus (Valenciennes, 1837) (Urbanski et al., 2020) y Hoplosternum littorale (Hancock, 1828) (Silva-Cavalcanti et al., 2017). En el Perú hasta el momento sólo se realizaron estudios principalmente en las playas de la zona costera (Purca \& Henostroza, 2017; Iannacone et al., 2019) y en peces marinos de las especies Odontesthes regia (Humboldt, 1821), Chirodactylus variegatus (Valenciennes, 1833) y Anisotremus scapularis (Tschudi, 1846) (De la Torre et al., 2019). Por tanto, la interacción de los microplásticos con los organismos de agua dulce y los riesgos asociados con el consumo humano de organismos que han ingerido microplásticos sigue siendo una importante laguna de conocimiento (Silva-Cavalcanti etal.,2017; Urbanski etal.,2020). En ese contexto, el presente estudio tuvo como objetivo investigar la presencia de microplásticos en diferentes especies de peces de importancia comercial, provenientes del mercado Belén, lugar frecuente del abastecimiento de alimentos para la población de la ciudad de Iquitos, en la región Loreto, Perú.

\section{MATERIAL Y MÉTODOS}

El lugar de colecta fue el Mercado Belén (zona baja) de la ciudad de Iquitos, región Loreto, Perú, en puestos de venta ambulatoria cercanos a las coordenadas 18M 694640 E y 9583805 S (Figura 1). Los 135 peces colectados en los meses de julio y agosto del año 2020 (período de vaciante) fueron de diferentes especies y de hábitos alimenticos detritívoros, omnívoros y 


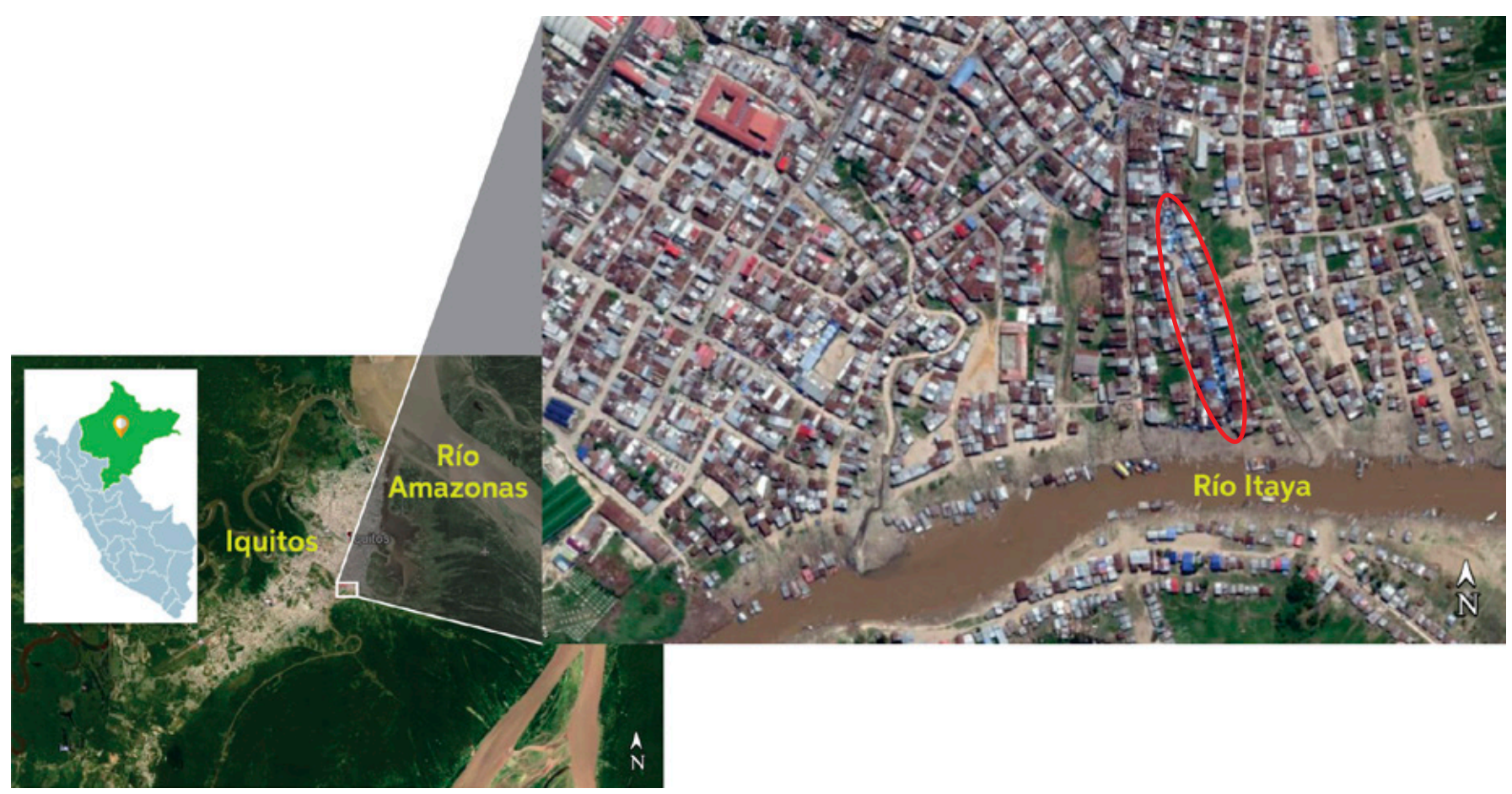

Figura 1. Ubicación de la zona de colecta de los peces, el Mercado Belén (elipse roja), perteneciente al distrito de Belén de la ciudad de lquitos, Perú (imágenes obtenidas de Google Earth Pro).

piscívoros. Los detritívoros fueron boquichico Prochilodus nigricans Agassiz, 1829 y llambina Potamorhina altamazonica (Cope, 1878); y los omnívoros fueron palometa Mylossoma albiscopum (Cope, 1872), sardina Triportheus angulatus (Spix \& Agassiz,1829), arahuana Osteoglossum bicirrhosum (Cuvier, 1829), lisa Schizodon fasciatus Spix \& Agassiz, 1829 y cunchi Pimelodus blochii Valenciennes, 1840. En tanto que, los piscívoros fueron huapeta Hydrolycus scomberoides (Cuvier, 1819), paña Pygocentrus nattereri Kner, 1858, mota Calophysus macropterus (Lichtenstein, 1819) y shiripira Sorubim lima (Bloch \& Schneider, 1801). La posible procedencia geográfica de los peces fue consultada a los vendedores para tenerlo como referencia. Por la cercanía de la zona de colecta, se compraron los peces más frescos posible y sin preservantes, estos fueron transportados inmediatamente al Laboratorio de Calidad de Agua de la Dirección de Investigación en Ecosistemas Acuáticos Amazónicos (AQUAREC) del Instituto de Investigaciones de la Amazonía Peruana (IIAP), para ser conservados a $4^{\circ} \mathrm{C}$ hasta su procesamiento.

En ellaboratorio se realizaron las mediciones de la longitud total $(\mathrm{cm})$ y el peso $(\mathrm{g})$ de los pescados. Posteriormente fueron lavados con agua destilada y luego con una tijera de disección se hizo un corte en la parte abdominal para extraer las vísceras y ubicar el estómago, los cuales fueron separados para la revisión de su contenido diluido en etanol filtrado al $70 \%$ en una placa petri (Urbanski et al., 2020). Los microplásticos fueron identificados con un estereoscopio trinocular Nikon, modelo SMZ800, al cual fue acoplada una cámara fotográfica Canon EOS Rebel T5i. Para el control de posibles contaminantes del ambiente de trabajo se utilizaron batas de laboratorio, guantes, gorros $\mathrm{y}$ todos los materiales de vidrio, metal y las 
superficies de trabajo fueron esterilizadas con etanol (Silva-Cavalcanti et al., 2017). Además, se pusieron dos placas Petri con etanol filtrado al $70 \%$ junto al estereoscopio (Silva-Cavalcanti et al., 2017; Urbanski et al., 2020), los cuales fueron revisados al final del trabajo con cada estómago (Silva-Cavalcanti et al., 2017; Andrade et al., 2019; Urbanski et al., 2020).

Para determinar si las partículas fueron sintéticas el método utilizado fue el visual, aplicando los principales criterios propuestos por Norén (2007) e Hidalgo-Ruz et al. (2012) para el tipo, forma y color de los microplásticos: 1) No se vean estructuras celulares u orgánicas en la partícula o fibra de plástico; 2) Si la partícula es una fibra, debe ser igualmente gruesa, no ahusada hacia los extremos y tener una flexión tridimensional (fibras no enteramente rectas que indica un origen biológico). 3) Partículas claras y de color homogéneo (azul, rojo, negro y amarillo). Es por ello que algunas partículas encontradas fueron descartadas por no cumplir con estas consideraciones, entre ellos las fibras transparentes y las blancas (por su similitud con antenas y fibras de diferentes organismos tanto animales, plantas y protistas). Esto fue importante para evitar la identificación errónea de los microplásticos, sobre todo cuando no es posible el uso de métodos más precisos tales como la Espectrofotometría infrarroja con transformada de Fourier FT-IR (Hidalgo-Ruz et al., 2012). Pero el aspecto metodológico más importante incluido fue que se descartaron las partículas que flotaron en el alcohol o se quedaron sobre el contenido estomacal, y sólo fueron considerados para el conteo los microplásticos encontrados dentro del mismo, para así minimizar la contaminación proveniente del ambiente de trabajo. Es decir, al igual que Norén (2017) las partículas se examinaron de forma conservadora, aunque la principal consecuencia de esto sea una subestimación de los microplásticos en los peces.

Los microplásticos fueron caracterizados en términos del color, la forma y el tamaño (SilvaCavalcanti et al., 2017; Urbanski et al., 2020). El tamaño fue estimado tomando en cuenta la mayor longitud de la partícula (Silva-Cavalcanti et al., 2017) con el programa Imagen J. La ingestión de microplásticos fue caracterizada por el número de partículas ingeridas por los peces (Silva-Cavalcanti et al., 2017) y el porcentaje de frecuencia de ocurrencia de microplásticos (FO\%= número de tractos gastrointestinales que contuvieron microplásticos / número total de tractos gastrointestinales analizados $\mathrm{x}$ 100) (Silva-Cavalcanti et al., 2017; Pegado et al., 2018; Urbanski etal.,2020). Las estadísticas descriptivas (media y desviación estándar) fueron realizadas con el programa Sigma Plot 11.0.

\section{RESULTADOS}

De los 135 individuos colectados 50 presentaron contenido estomacal y fueron incluidos en el estudio. En los detritívoros fueron analizados 13 individuos de $P$. nigricans y tres individuos de $P$. altamazonica. En los omnívoros fueron revisados cuatro individuos de M. albiscopum, 10 individuos de $T$. angulatus, un individuo de 0 . bicirrhosum, ocho individuos de $S$. fasciatus y un individuo de P. blochii. En tanto que, en los piscívoros se analizaron cuatro individuos de H. scomberoides, un individuo de $P$. nattereri, cuatro individuos de C. macropterus y un individuo de S. lima. En la Tabla 1 se muestran las longitudes totales y los pesos de las especies estudiadas.

De todos los peces analizados, sólo seis individuos de P. nigricans (12\% de los analizados) presentaron microplásticos en el contenido estomacal (Tabla 2). En las especies P. altamazonica y C. macropterus, también fueron identificadas 
Tabla 1: Número de individuos, longitud total y peso de las especies de peces estudiadas provenientes del Mercado Belén, lquitos-Perú.

\begin{tabular}{|c|c|c|c|c|c|}
\hline \multirow[t]{2}{*}{ Especie } & \multirow[t]{2}{*}{$\begin{array}{c}\mathrm{N}^{\circ} \text { de } \\
\text { Individuos }\end{array}$} & \multicolumn{2}{|c|}{$\begin{array}{l}\text { Longitud Total } \\
\qquad(\mathrm{cm})\end{array}$} & \multicolumn{2}{|r|}{$\begin{array}{l}\text { Peso } \\
(\mathrm{g})\end{array}$} \\
\hline & & Rango & Media \pm Des. Est. & Rango & Media \pm Des. Est. \\
\hline Prochilodus nigricans & 13 & $18-25$ & $20,7 \pm 1,9$ & $91-165$ & $113,4 \pm 22,2$ \\
\hline Potamorhina altamazonica & 3 & $18,3-20,8$ & $19,4 \pm 1,3$ & $98-120$ & $109,7 \pm 11,1$ \\
\hline Mylossoma albiscopum & 4 & $15,7-17$ & $16,4 \pm 0,5$ & $86-100$ & $94 \pm 7,1$ \\
\hline Triportheus angulatus & 10 & $16,5-18,9$ & $17,7 \pm 0,8$ & $48-71$ & $62,1 \pm 7,3$ \\
\hline Osteoglossum bicirrhosum & 1 & 39.3 & - & 280 & - \\
\hline Schizodon fasciatus & 8 & $22,5-27,1$ & $24,7 \pm 1,6$ & $109-178$ & $146,9 \pm 25,1$ \\
\hline Pimelodus blochii & 1 & & - & & - \\
\hline Hydrolycus scomberoides & 4 & $26,3-28,7$ & $27,5 \pm 1,2$ & $121-185$ & $205,8 \pm 33,7$ \\
\hline Pygocentrus nattereri & 1 & 16 & - & 78 & - \\
\hline Calophysus macropterus & 4 & $31,5-43,8$ & $35 \pm 5,9$ & $211-728$ & $371 \pm 239,9$ \\
\hline Sorubim lima & 1 & 28,5 & - & 125 & - \\
\hline
\end{tabular}

Tabla 2: Cantidad, forma y color de los microplásticos identificados en seis individuos de Prochilodus nigricans Agassiz, 1829 colectados en el Mercado Belén, lquitos-Perú.

\begin{tabular}{cccc}
\hline $\mathbf{N}^{\circ}$ de Individuo & $\begin{array}{c}\text { Número de } \\
\text { Microplásticos }\end{array}$ & Formas (Número) & Colores (Número) \\
\hline 1 & 1 & Filamento & Azul \\
\hline 2 & 1 & Filamento & Azul \\
\hline 3 & 2 & Filamento & Rojo \\
\hline 4 & 2 & Filamento, Fragmento Rojo \\
\hline 5 & 4 & Filamento (2), Fragmento (2) & Azul (3), Celeste (1) \\
\hline 6 & 7 & Filamento & Azul (4), Rojo (2), Verde (1) \\
\hline TOTAL & 17 & & \\
\hline
\end{tabular}


algunas partículas, pero por ser transparentes y/o blancas no fueron tomadas en cuenta. En total fueron identificadas 17 partículas en P. nigricans (Tabla 2), y todas estuvieron dentro del rango de tamaño de los microplásticos de 0,40 a 4,39 mm. El número de microplásticos por individuo fue de 1 , 2,4 y 7 , con un promedio (y desviación estándar) de $0,34( \pm 1,19)$ microplásticos por individuo. Las formas encontradas fueron los filamentos y los fragmentos irregulares (Figura 2, Tabla 2), siendo los filamentos los más abundantes con 14 microplásticos $(82,4 \%$ de los encontrados). Los colores identificados fueron el azul, rojo, verde y celeste (Figura 2, Tabla 2), siendo los azules los más abundantes con 10 microplásticos (58,8\% de los identificados). La frecuencia de ocurrencia de microplásticos para los 50 individuos fue de $12 \%$ y para $P$. nigricans fue de $46,2 \%$.
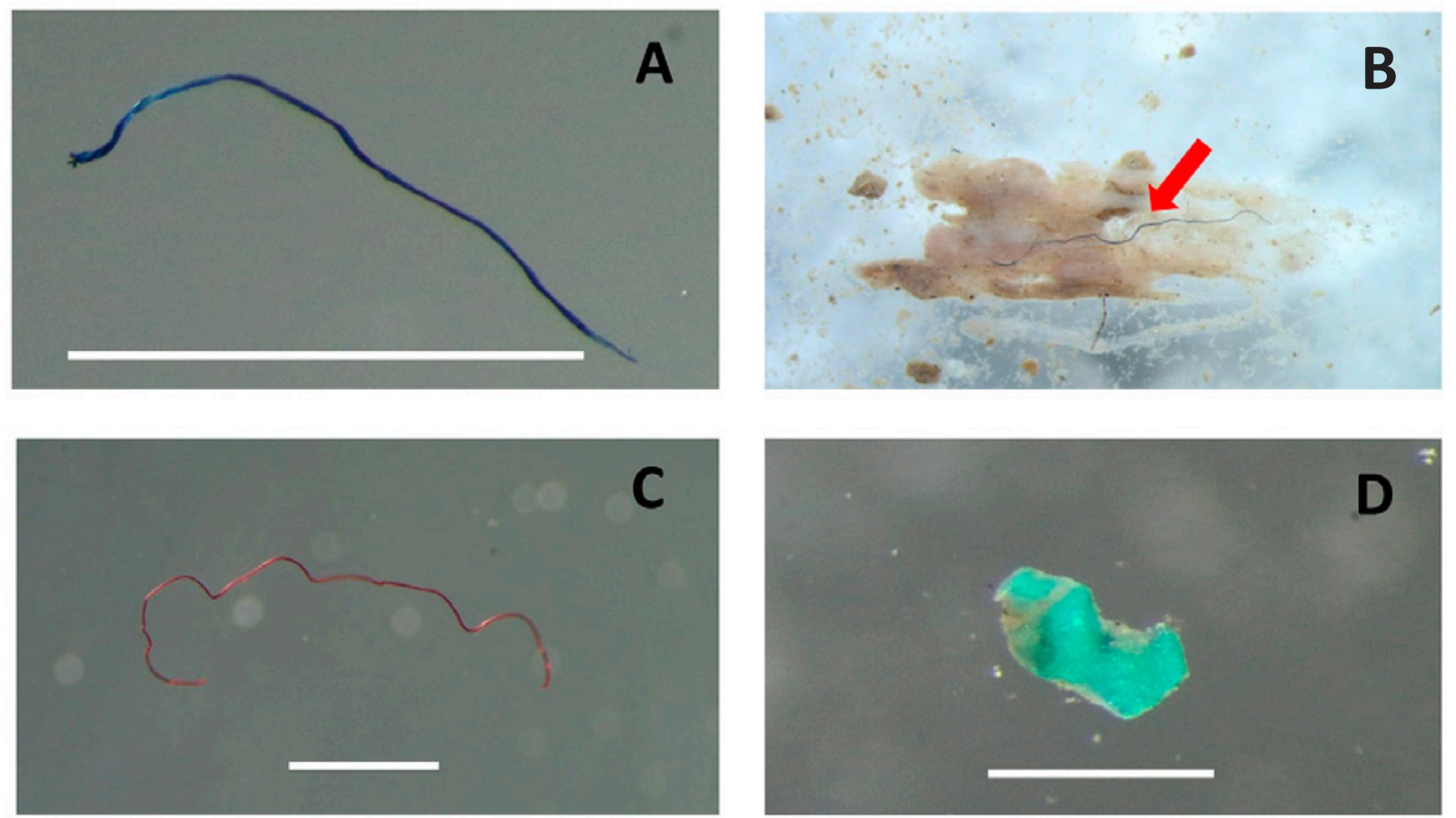

Figura 2: Microplásticos encontrados en los estómagos del boquichico Prochilodus nigricans provenientes del mercado Belén de la ciudad de lquitos, Perú. Las fotos A, B y C son ejemplos de filamentos y el D es un ejemplo de fragmento irregular. Las fotos A y B muestran microplásticos de color azul, la foto C el rojo y la foto D el verde. La foto B muestra un microplástico azúl dentro del contenido estomacal (flecha roja). Barra de escala = $1 \mathrm{~mm}$. 
una comprensión holística de los factores que influyen (Roch et al., 2020).

Si bien el número de partículas identificadas en P. nigricans fue baja en relación a otros estudios realizados en peces tropicales (Silva-Cavalcanti et al., 2017; Pegado et al., 2018; Andrade et al., 2019; Urbanski et al., 2020), la ingestión pudo deberse a la absorción accidental durante la búsqueda de alimento. Por ser una especie iliófaga, se alimenta de detritos orgánicos, microorganismos y materia orgánica en descomposición, generalmente depositados en el fondo de los ríos (García-Dávila et al., 2018; Urbanski et al., 2020). Esta vía ya fue discutida para Prochilodus lineatus, otra especie iliófaga donde se encontraron microplásticos en la cuenca del río Tietê (sureste de Brasil), proponiendo que los sedimentos podrían ser la fuente de contaminación (Urbanski et al., 2020). Asimismo, en varios estudios alrededor del mundo verificaron el predominio del color azul entre los plásticos ingeridos, proponiendo que las diferentes especies de peces pueden tener predilección por ese color o que la mayor parte de la contaminación plástica está compuesta por material azul (Urbanski et al., 2020). Esta segunda posibilidad también parece explicar el mayor número de microplásticos azules reportados para $P$. nigricans, toda vez que al igual $P$. lineatus se alimenta de los sedimentos del fondo, donde la discriminación de los alimentos por color es difícil debido a la poca penetración de la luz (Urbanski et al., 2020). Por este motivo, la caracterización de la deposición de partículas de plástico en la columna de agua y sedimentos es una necesidad de investigación para una mejor comprensión de este problema ambiental (Urbanski et al., 2020), y con los resultados actuales obtenidos se hace imperativo en los ecosistemas acuáticos de la Amazonía peruana.

El primer registro de microplásticos en peces amazónicos fue en especies de pirañas y otros serrasalmidos (Andrade et al., 2019), pero el registro en $P$. nigricans es muy importante debido a que es una especie de importancia nutricional y comercial en la Amazonía (Silva \& Stewart, 2017), que ocupa los primeros lugares en desembarques pesqueros (García et al., 2009; García et al., 2012; García-Dávila et al., 2018). Los microplásticos se sumarían a otras perturbaciones antropogénicas al que está sometida P. nigricans actualmente, como son la actividad petrolera (se encontraron evidencias de exposición hasta en los otolitos) y por ser especie migratoria viene siendo afectada las hidroeléctricas (Silva \& Stewart, 2017). En otras especies como la perca europea Perca fluviatilis Linnaeus, 1758 se ha demostrado que la exposición a relevantes concentraciones de microplásticos de poliestireno inhibe la eclosión, disminuye las tasas de crecimiento, altera las preferencias alimentarias y el comportamiento innato (Lönnstedt \& Eklöv, 2016).

Los resultados sugieren también que algunas zonas de pesca en la Amazonía peruana están siendo impactadas por la mala disposición de residuos sólidos. En el caso específico de los peces analizados, pudieron provenir de zonas cercanas a Iquitos (los peces fueron frescos); pudiendo incluso ser de la parte baja del rio Itaya (justo a la altura del mercado Belén). Esta zona es una de las más impactadas por la contaminación por residuos sólidos (Faldetta et al., 2014; Ayllón \& Pérez, 2015; Ramirez, 2016), y donde frecuentemente se observan a personas realizando pesca (Ayllón \& Pérez, 2015; Chota-Macuyama obs. pers.). En los próximos estudios podremos corroborar este hecho, haciendo comparaciones entre cuencas o zonas con diferentes niveles de impacto de residuos sólidos.

\section{AGRADECIMIENTOS}

Agradecemos a la Oficina General de Administración del Instituto de Investigaciones de la 
Amazonia Peruana (IIAP) por la gestión adecuada de los fondos institucionales, el cual permitió el desarrollo de la investigación. Asimismo, agradecemos a los revisores por las observaciones realizadas.

\section{REFERENCIAS BIBLIOGRÁFICAS}

Andrade, M.C.; Kirk O. Winemiller, K.O.; Barbosa, P.S.; Fortunati, A.; Chelazzi, D.; Cincinelli, A.; Giarrizzo, T. 2019. First account of plastic pollution impacting freshwater fishes in the Amazon: Ingestion of plastic debris by piranhas and other serrasalmids with diverse feeding habits. Environmental pollution, 244: 766-773. DOI: https://doi.org/10.1016/j. envpol.2018.10.088

Ayllón, Z; Pérez, M. 2015. Contaminación del agua del río Itaya por agentes biológicos patógenos y su impacto en la salud humana. Tesis de Maestría, Universidad Nacional de la Amazonía Peruana, Iquitos, Perú. 141pp.

Cole, M.; Lindeque, P.; Halsband, C.; Galloway, T.S. 2011. Microplastics as contaminants in the marine environment: a review. Marine Pollution Bulletin, 62: 2588-2597. DOI: https://doi. org/10.1016/j.marpolbul.2011.09.025

De La Torre, G.E.; Dioses-Salinas, D.C.; Pérez-Baca, B.L.; Santillán, L. 2019. Microplastic abundance in three commercial fish from the coast of Lima, Peru. Brazilian Journal of Natural Sciences, 2(3): 171-177. DOI: https://doi.org/10.31415/bjns. v2i2.67

De Sales-Ribeiro, C.; Brito-Casillas, Y.; Fernandez, A.; Caballero, M.J. 2020. An end to the controversy over the microscopic detection and efects of pristine microplastics in fish organs. Scientific Reports, 10: 12434. DOI: https://doi. org/10.1038/s41598-020-69062-3

Faldetta, K.F.; Reighard, D.A.; Dickinson, K.L.; Wang, C.Q.; George, D.R.; Rodriguez, L.; Strosnider,
W.H.J. .2014. Assessing domestic water quality in Belén municipality, Iquitos, Peru. Journal of Water, Sanitation and Hygiene for Development, 4(3): 391-399. DOI: https://doi.org/10.2166/ washdev.2014.051

Frias, J.P.G.L.; Nash, R. 2019. Microplastics: Finding a consensus on the definition. Marine Pollution Bulletin, 138: 145-147. DOI: https:// doi.org/10.1016/j.marpolbul.2018.11.022

García, A.; Tello, S.; Vargas, G.; Duponchelle, F. 2009. Patterns of commercial fish landings in the Loreto region (Peruvian Amazon) between 1984 and 2006. Fish Physiology and Biochemistry, 35: 53-67. DOI: https://doi. org/10.1007/s10695-008-9212-7

García, A.; Tello, S.; Vargas, G.; Duponchelle, F. 2012. Desembarque de pescado fresco en la ciudad de Iquitos, región Loreto-Amazonia peruana. Folia Amazónica, 21(1-2): 45-52. DOI: https://doi.org/10.24841/fa.v21i1-2.31

García-Dávila, C.; Sánchez, H.; Flores, M.; Mejia, J.; Angulo, C.; Castro-Ruiz, D.; Estivals, G.; García, A.; Vargas, G.; Nolorbe, C.; Núñez, J.; Mariac, C.; Duponchelle, F.; Renno, J.F. 2018. Peces de consumo de la Amazonía peruana. Instituto de Investigaciones de la Amazonía Peruana (IIAP). Iquitos, Perú. 218pp.

Hidalgo-Ruz, V.; Gutow, L.; Thompson, R.C.; Thiel, M. 2012. Microplastics in the marine environment: A review of the methods used for identification and quantification. Environmental Science \& Technology, 46: 3060-3075. DOI: https://doi.org/10.1021/es2031505

Iannacone, J.; Huayhua, A.; Alvariño, L.; Valencia, F.; Principe, F.; Minaya, D.; Ortega, J.; Argota, G.; Castañeda, L. 2019. Microplásticos en la zona de marea alta y supralitoral de una playa arenosa del litoral costero del Perú. The Biologist, 17(2): 335-346. DOI: http://dx.doi.org/10.24039/ rtb2019172369

Lönnstedt, O.M.; Eklöv, P. 2016. Environmentally relevant concentrations of microplastic 
particles influence larval fish ecology. Science, 352(6290): 1213-1216. DOI: https://doi.org/ 10.1126/science.aad8828

Lusher, A.L.; Hollman, P.C.H.; Mendoza-Hill, J.J. 2017. Microplastics in fisheries and aquaculture: status of knowledge on their occurrence and implications for aquatic organisms and food safety. Food and Agriculture Organization of the United Nations (FAO). Fisheries and Aquaculture Technical Paper, 615. Rome, Italy. 126pp.

Norén, F. 2007. Small plastic particles in coastal swedish waters. KIMO Sweden. Lysekil, Suecia. 11pp.

Pegado, T.; Schmid, K.; Winemiller, K.; Chelazzi, D.; Cincinelli, A.; Dei, L.; Giarrizzo, T. 2018. First evidence of microplastic ingestion by fishes from the Amazon river estuary. Marine Pollution Bulletin, 133: 814-821. DOI: https:// doi.org/10.1016/j.marpolbul.2018.06.035

Purca, S.; Henostroza, A. 2017. Presencia de microplásticos en cuatro playas arenosas de Perú. Revista Peruana de Biología, 24(1): 101106. DOI: http://dx.doi.org/10.15381/rpb.v24 i1.12724

Ramirez, P. 2016. Identificación de las fuentes de contaminación y su relación con la dinámica del río Itaya (zona baja de Belén), distrito de
Belén. 2014. Tesis de pre-grado, Universidad Nacional de la Amazonia Peruana, Facultad de Agronomía, Iquitos, Perú. 70pp.

Roch, S.; Friedrich, C.; Brinker, A. 2020. Uptake routes of microplastics in fishes: practical and theoretical approaches to test existing theories. Scientific Reports, 10: 3896. DOI: https://doi. org/10.1038/s41598-020-60630-1

Silva-Cavalcanti, J.; Silva, J.D.B.; De França, E.J.; Barbosa de Araújo, M.C.; Gusmão, F. 2017. Microplastics ingestion by a common tropical freshwater fishing resource. Environmental Pollution, 221: 218-226. DOI: http://dx.doi. org/10.1016/j.envpol.2016.11.068

Silva, E.; Stewart, D.J. 2017. Reproduction, feeding and migration patterns of Prochilodus nigricans (Characiformes: Prochilodontidae) in northeastern Ecuador. Neotropical Ichthyology, 15(3): e160171. DOI: http://dx. doi.org/ 10.1590/1982-0224-20160171

Urbanski, B.Q.; Denadai, A.C.; Azevedo-Santos, V.M.; Nogueira, M.G. 2020. First record of plastic ingestion by an important commercial native fish (Prochilodus lineatus) in the middle Tietê river basin, Southeast Brazil. Biota Neotropica, 20(3): e20201005. DOI: https:// doi.org/10.1590/1676-0611-BN-2020-1005

Recibido: 14 de octubre de 2020 Aceptado para publicación: 18 de diciembre de 2020 